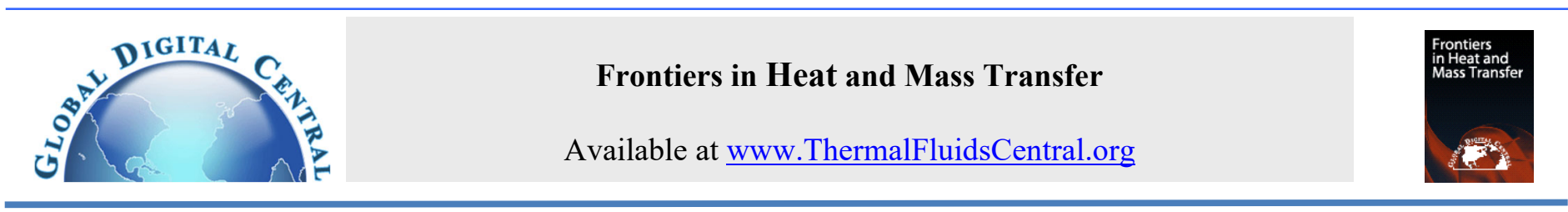

\title{
ANALYSIS OF ARRHENIUS ACTIVATION ENERGY IN ELECTRICALLY CONDUCTING CASSON FLUID FLOW INDUCED DUE TO PERMEABLE ELONGATED SHEET WITH CHEMICAL REACTION AND VISCOUS DISSIPATION
}

\author{
N. Vijaya ${ }^{\mathrm{a},}$, S. M. Arifuzzaman ${ }^{\mathrm{b}}$, N. Raghavendra Sai ${ }^{\mathrm{c}}$, Ch. Manikya Rao ${ }^{\mathrm{d}}$ \\ ${ }^{a}$ Department of Mathematics, Koneru Lakshmaiah Education Foundation, Vaddeswaram, Guntur, AP, India - 522502. \\ ${ }^{b}$ Centre for Infrastructure Engineering, Western Sydney University, NSW, 2751, Australia. \\ ${ }^{c}$ Department of Computer Science and Engineering, Koneru Lakshmaiah Education Foundation, Vaddeswaram, Guntur, AP, India- 522302. \\ ${ }^{d}$ Department of Mathematics, Bapatla College of Arts \& Science, Bapatla, A.P, India-522101.
}

\begin{abstract}
The upfront intension of this study is to explore the advances in electrically conducting Casson fluid induced due to a porous elongated surface taking Arrhenius activation energy, viscous dissipation and joule heating into account. Uniform magnetic and electric fields are imposed on the given flow. Variables of similarity are induced to transmute partial differential equations into dimensionless equations and resolved numerically by elegant method bvp4c. To scrutinize the behavior of critical parameters on flow configurations graphs and table are portrayed. From graphical moments, it is analyzed that velocity of the liquid diminish for advanced values of non-Newtonian rheology parameter, magnetic parameter, porous parameter and inertial parameter. This study also reported that activation energy parameter enhances concentration profiles, whereas fitted rate constant shows opposite behavior. Impact of skin friction, Sherwood and Nusselt numbers on the flow configurations for diverse critical parameters are exposed realistically via graphs.

Key words: Non Newtonian Rheology parameter, porous parameter, magnetic parameter, Casson constitutive equations, Arrhenius equation, activation energy parameter, reaction rate parameter, electrical field parameter.
\end{abstract}

\section{INTRODUCTION}

Chemical reaction plays dynamic role in many industrial and technological applications, so there is a great demand to minimize number of reagents and to maximize desired output. Solar energy became the alternative energy source all over the world. But solar technologies have many obstacles like low lifetime of solar cell, small execution balance of structures. Deep insight of activation energy on these sources is helpful to overcome impediments and enlightening the energy stability. The Arrhenius activation energy combined with chemical reaction in MHD flows has countless applications such as chemical engineering, geology, oil refineries, food industries, lubricants so forth. This energy of activation is the quantity of energy that is prerequisite to break down the chemical bonds to start up the chemical reaction. Arrhenius equation best explains transport phenomena under chemical reaction and temperature. Zeeshan et al. (2018) explains the influence of activation energy in Couette Poiseuille of nano fluid. Irfan et al. (2019) studied combined effect of both these energies in 3D Carreau nano fluid by inducing Buongiorno's theory. Activation energy in peristalsis of Jeffrey fluid is explored by Hayat $e t$ al. (2019). Arifuzzaman et al. (2018, 2019) implemented finite difference method to explore influence of chemical reaction on $4^{\text {th }}$ and $2^{\text {nd }}$ grade fluid transport property and MHD flow past porous plates. Hosia (2017) promoted radiation electrical MHD activation energy using Carreau- Nanofluid with parameters control method. Reza-ERabbi et al. (2020) enlightened chemical retort on Casson fluid past an elongated sheet using finite difference method and nonlinear chemical reaction effects on multiphase Casson fluid.

The viscosity of the fluid like paints, greases, lubricant oils coal tar, jellies, and paste is not fixed and it depends upon the factors like shear in fluid, pressure and temperature. These fluids are known as non-Newtonian fluids in nature. Casson fluid is non-Newtonian and first proposed by enthusiastic Mathematician Casson (1959) while doing his experiments on letter press toners. The special properties of Casson fluid passed significant applications in science as well as in polymer processing and in biomechanics. Humanoid blood can be measured as Casson fluid because it contains protein, fibrinogen, etc. Inducing Laplace transform technique Hari et al. (2018) obtained exact and numerical solution for unsteady convective Casson fluid considering magnetic field into account. Fazal Mabood et al. (2019) focused porous medium in Casson fluid flow under thermal radiation.

Sulochana et al. (2019) elaborately explained hall current result in Casson fluid flow Vijaya et al. (2018, 2019, 2020) comprehensively studied Casson fluid and Casson Nanofluid on stretching surface by considering the impact of radiation, thermophorosis, chemical reaction and Brownian motion. Gayetri et al. (2020) considered Carreau fluid through a stretching sheet with variable thickness and observes that Weissenberg number boosts the velocity. Naga Santhoshi et al. (2020) deliberately discussed three dimensional Casson and Carreau

*Corresponding author. Email: vijayanalleboyina@gmail.com 
nanofluid flow configurations taking convective boundary conditions into account. Konda Jaya rami reddy et al. (2018) elevated properties of Casson nanofluid past a nonlinear permeable stretching sheet with different physical and boundary conditions. Dharmaiah et al. (2018) explored MHD fluid flow over a semi-infinite flat plate with absorption and heat source.

MHD flow intensifies the curiosity of many researchers now a days for the reason that it plays big role in numerous applications in all fields of science and technology like plasma physics, aerodynamics, astrophysics and super conduction coils. The physical phenomena of viscous flows through porous media can be observed in energy extraction from thermal regions, solid filtration, sedimentary petrology, and also blood stream in the tissue region. MHD flow of Carreau Nano fluid explored using CNT over a nonlinear elongated sheet is studied by Nagalakshmi et al. (2020). Dhana Lakshmi et al. (2019) studied porous effects on MHD convective flows. Sandya et al. (2019) explore MHD flow of inclined porous plates under the influence of radiation and chemical reaction. Kumar et al. (2019) considered slip flow regime with chemical effects in unsteady MHD flow. Vedhavathi et al. (2017) examined combined effects of chemical reaction and radiation of a nanofluid bounded by semi-infinite flat plate. Micropolar fluid over a stretching sheet in porous medium under the influence of radiation and chemical rection is studied by Charan kumar et al. (2018). Chandra sekhar et al. (2018) studied second grade fluid over a stretching sheet and MHD free convective heat transfer flows under different conditions. Krishna et al. (2018) studied chemical reaction effects of MHD Casson fluid flow. Sateesh kumar et al. (2019) eloborately discussed the properties of visco elastic fluid between two porous plates. Ramana murthy et al. (2019) applied Mathematical model for increasing incidence of tuberculosis in poverty driven confirmed areas. Radhamadhavi et al. (2019) studied mixed convective nanofluid over vertical circular cylinder by considering physical conditions like magnetic field, heat radiation, and surface temparature.

The major objective of this study is to explore binary chemical reaction along with activation energy of an electrically conducting incompressible Casson fluid induced due to stretching surface. The aspects of viscous and Joule heating are also incorporated. Graphical portrayal is depicted using the powerful tactic bvp4c via MATLAB software.

\section{PHYSICAL MODEL}

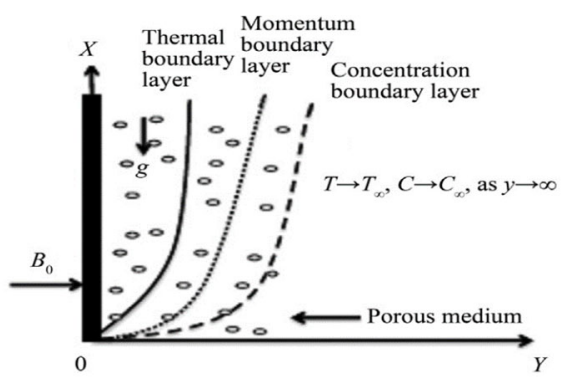

Fig. 1 Geometrical outline of the flow

A two dimensional incompressible steady Darcy-Forchheimer mixed convective electrically conducting Casson fluid flow induced by a vertically elongated sheet inserted in a fluid saturated porous medium is presented in this study. In this flow geometry, origin is taken as the fixed point and the pane is elongated along $\mathrm{x}$-axis as well as y-axis. Two identical and reverse forces are applied to stretch the sheet. Maxwell's equation $\nabla \cdot \vec{B}=0, \nabla \times \vec{E}=0$, and Ohm's law $\vec{J}=$ $\sigma(\vec{E}+\vec{q} \times \vec{B})$ are measured in the precise flow, with $\vec{B}, \vec{E}, \vec{J}, \sigma, \vec{q}$ are transverse magnetic field, transverse electric field, Joule current, magnetic permeability and fluid velocity respectively. The very slight induced magnetic field generated due to electrical fluid is ignored in this study.

Casson fluid is non-Newtonian in nature and its constitutive equations (Eldabe and Salwa, 1995) are written as follows.

$$
\begin{aligned}
& \text { for } \pi>\pi_{c}, \tau_{\mathrm{ij}}=2\left(\mu_{\mathrm{B}}+\mathrm{P}_{\mathrm{y}}(2 \pi)^{\frac{-1}{2}}\right) \mathrm{e}_{\mathrm{ij}} \\
& \text { for } \pi<\pi_{c} \cdot \tau_{\mathrm{ij}}=2\left(\mu_{\mathrm{B}}+\mathrm{P}_{\mathrm{y}}\left(2 \pi_{C}\right)^{\frac{-1}{2}}\right) \mathrm{e}_{\mathrm{ij}}
\end{aligned}
$$

In the above constitutive equations $\tau_{i j}$ is the $(i, j)^{\text {th }}$ stress tensor component, $\mu_{\mathrm{B}}$ is the plastic dynamic viscosity of the fluid which is non-Newtonian. When $\mathrm{P}_{\mathrm{y}}>\mathrm{P}_{\mathrm{s}}$ fluid acts as a solid, and when $\mathrm{P}_{\mathrm{y}}<\mathrm{P}_{\mathrm{s}}$ fluid demonstrates flow characteristics, where $P_{y}$ is yield stress, $P_{s}$ is shear stress. $\pi_{c}$ is the critical value of $\pi=e_{i j} e_{i j}\left(e_{i j}\right.$ is the $(i, j)^{\text {th }}$ component of deformation rate) which depends upon non-Newtonian model. The governing equations of the flow are as prearranged here under with terms mentioned in nomenclature.

$$
\begin{aligned}
& \frac{\partial \bar{u}}{\partial x}+\frac{\partial \bar{v}}{\partial y}=0 \\
& \bar{u} \frac{\partial \bar{u}}{\partial x}+\bar{v} \frac{\partial \bar{u}}{\partial y}=v\left(1+\frac{1}{\beta}\right) \frac{\partial^{2} \bar{u}}{\partial y^{2}}+\frac{\sigma}{\rho}\left(E_{0} B_{0}-B_{0}^{2} \bar{u}\right)-\frac{C_{b}}{\sqrt{k_{p}}} \bar{u}^{2} \\
& -\frac{v}{k_{p}} \bar{u}+g \beta_{T}\left(T^{*}-T_{\infty}\right)+g \beta_{C}\left(C^{*}-C_{\infty}\right) \\
& \overline{\boldsymbol{u}} \frac{\partial \boldsymbol{T}^{*}}{\partial \boldsymbol{x}}+\overline{\boldsymbol{v}} \frac{\partial \boldsymbol{T}^{*}}{\partial \boldsymbol{y}}=\frac{\boldsymbol{k}}{\boldsymbol{\rho} \boldsymbol{c}_{\boldsymbol{p}}} \frac{\partial^{2} \boldsymbol{T}^{*}}{\partial \boldsymbol{y}^{\mathbf{2}}}+\frac{\mathbf{1 6} \boldsymbol{\sigma}^{*} \boldsymbol{T}_{\infty}^{3}}{3 \boldsymbol{k}^{*} \boldsymbol{\rho} \boldsymbol{c}_{\boldsymbol{p}}}+\frac{\boldsymbol{\mu}}{\boldsymbol{\rho} \boldsymbol{c}_{\boldsymbol{p}}}\left(\mathbf{1}+\frac{\mathbf{1}}{\boldsymbol{\beta}}\right)\left(\frac{\partial \overline{\boldsymbol{u}}}{\partial \boldsymbol{y}}\right)^{\mathbf{2}} \\
& +\frac{\boldsymbol{\sigma}}{\boldsymbol{\rho} \boldsymbol{c}_{\boldsymbol{p}}}\left(\overline{\boldsymbol{u}} \boldsymbol{B}_{\mathbf{0}}-\boldsymbol{E}_{\mathbf{0}}\right)^{\mathbf{2}} \\
& \bar{u} \frac{\partial C^{*}}{\partial x}+\bar{v} \frac{\partial C^{*}}{\partial y}=D \frac{\partial^{2} C^{*}}{\partial y^{2}}+\frac{D k_{T^{*}}}{T_{m}} \frac{\partial^{2} T^{*}}{\partial y^{2}}-k_{r}^{2}\left(C^{*}-C_{\infty}\right)\left(\frac{T^{*}}{T_{\infty}}\right)^{n} e^{\left(-\frac{E a}{\xi T^{*}}\right)} \\
& (5)
\end{aligned}
$$

(5)

In the above governing equations (3\&4), $\beta\left(=\mu_{\mathrm{B}} \sqrt{2 \pi_{\mathrm{c}}} / \mathrm{P}_{\mathrm{y}}\right)$ is the Casson parameter, and in equation (5) the term $k_{r}^{2}\left(\frac{T^{*}}{T_{\infty}}\right)^{n} e^{\left(-\frac{E a}{\xi T^{*}}\right)}$ represents the modified Arrhenius equation in which the reaction rate is given by $k_{r}^{2}$, the activation energy is given by $E_{a}$, the Boltzmann constant

is given by $\xi=8.61 \times 10^{-5} \mathrm{eV} / K$, and the fitted rate constant is given by ' $n$ ' which lies between -1 and 1 . Soret effect is also studied through

the term $\frac{D k_{T^{*}}}{T_{m}} \frac{\partial^{2} T^{*}}{\partial y^{2}}$ in the equation (5). The associated boundary conditions with stretching surface velocity is $u_{w}(x)=b x, T_{w}>$ $T_{\infty}, C_{w}>C_{\infty}$ where proportionality constant ' $\mathrm{b}$ ' are as follows.

$\left\{\begin{array}{c}\bar{u}=u_{w}(x) \\ \bar{v}=0 \\ T^{*}=T_{\infty} \\ C^{*}=C_{\infty}\end{array}\right.$ at $\mathrm{y}=0,\left\{\begin{aligned} u & \rightarrow 0 \\ T^{*} & \rightarrow T_{\infty} \\ C^{*} & \rightarrow C_{\infty}\end{aligned}\right.$ as $y \rightarrow \infty$

\section{SUITABLE CONVERSION}

Following likeness variables are introduced to transform PDE (2-5) into ODE

$\bar{u}=b x f^{\prime}(\eta), \bar{v}=-\sqrt{b v} f(\eta), \eta=\sqrt{\frac{b}{v}} y$ 
$\theta(\eta)=\frac{T^{*}-T_{\infty}}{T_{w}-T_{\infty}}, \quad \phi(\eta)=\frac{C^{*}-C_{\infty}}{C_{w}-C_{\infty}}$

Substituting the likeness variables defined in equation $(7 \& 8)$ into the governing equations (2) - (5) we obtain

$\left(1+\frac{1}{\beta}\right) f^{\prime \prime \prime}+f f^{\prime \prime}-f^{2}+M\left(E_{1}-f^{\prime}\right)-F_{r} f^{2}-D f^{\prime}+$
$R(\theta+N \phi)=0$

$\left(1+\frac{4}{3} N r\right) \theta^{\prime \prime}+\operatorname{Pr}\left[\begin{array}{c}f \theta^{\prime}+\operatorname{MEc}\left(E_{1}-f^{\prime}\right)^{2}+ \\ \left(1+\frac{1}{\beta}\right) E c\left(f^{\prime \prime}\right)^{2}\end{array}\right]=0$

$\phi^{\prime \prime}+\operatorname{Sc}\left(f \phi^{\prime}+\operatorname{Sr} \theta^{\prime \prime}\right)-\operatorname{Sc} \Lambda(1+\delta \theta)^{n} \phi e^{\left(-\frac{E}{1+\delta \theta}\right)}=0$

$\left\{\begin{array}{l}f(0)=0 \\ f^{\prime}(0)=1 \\ \theta(0)=1 \\ \phi(0)=1\end{array}\right.$ at $\eta=0 \quad\left\{\begin{array}{r}f^{\prime}(\infty)=0 \\ \theta(\infty)=0 \quad \text { as } \eta \rightarrow \infty, \\ \phi(\infty)=0\end{array}\right.$

Here $\mathrm{M}\left(=\frac{\sigma B_{0}^{2}}{\rho b}\right)$ the magnetic parameter, $\mathrm{R}\left(=\frac{G r_{x}}{R e_{x}^{2}}\right)$ the thermal buoyancy number, $\mathrm{N}\left(=\frac{G c}{G r_{x}}\right)$ the solutal buoyancy number, $G r_{x}(=$ $\left.\frac{g \beta_{T}\left(T_{w}-T_{\infty}\right) x^{3}}{v^{2}}\right)$ the Grashof number due to temperature, Gc $(=$ $\left.\frac{g \beta_{C}\left(C_{w}-C_{\infty}\right) x^{3}}{v^{2}}\right)$ the Grashof number due to concentration, $E_{1}\left(=\frac{E_{0}}{B_{0} b x}\right)$ the electrical field parameter, $F_{r}\left(=\frac{C_{b} x}{\sqrt{k_{1}}}\right)$ the inertial parameter, D $\left(=\frac{v}{k_{p} b}\right)$ the permeability parameter, $\operatorname{Pr}\left(=\frac{\rho c_{p} v}{k}\right)$ the Prandtl number, $N r\left(=\frac{4 \sigma^{*} T_{\infty}^{3}}{k k^{*}}\right)$ the thermal radiation parameter, $E c\left(=\frac{U_{w}{ }^{2}}{c_{p}\left(T_{w}-T_{\infty}\right)}\right)$ the Eckert number, $\operatorname{Sc}\left(=\frac{v}{D}\right)$ the Schmitd's number, $\operatorname{Sr}\left(=\frac{D k_{T}\left(T_{w}-T_{\infty}\right)}{v T_{m}\left(C_{w}-C_{\infty}\right)}\right)$ the Soret number, $\Lambda\left(=\frac{k_{r}^{2}}{a}\right)$ the reaction rate parameter, $\mathrm{E}\left(=\frac{E_{a}}{\xi T_{\infty}}\right)$ the activation energy parameter, $\delta\left(=\frac{T_{w}-T_{\infty}}{T_{\infty}}\right)$ the temperature difference parameter.

\section{PHYSICAL QUANTITIES}

The significant engineering physical quantities in this problem are $C_{f}$ (=skin friction coefficient), $N u_{x}$ (=local Nusselt number), $S h_{x}$ (=local Sherwood number) respectively are defined below with $R e_{x}=$ $\frac{u_{w} x}{v}$ as local Reynolds number

$C_{f}=\frac{2 \tau_{w}}{\rho u_{w}^{2}}, \quad N u_{x}=\frac{x q_{w}}{K\left(T_{w}-T_{\infty}\right)}$ and $S h_{x}=\frac{x q_{m}}{D_{m}\left(C_{w}-C_{\infty}\right)}$

$\tau_{w}, q_{w}, q_{m}$ are wall shear stress, heat transfer, mass transfer respectively and are given by

$\tau_{w}=\mu\left(1+\frac{1}{\beta}\right)\left(\frac{\partial u}{\partial y}\right)_{y=0}$

$q_{w}=-K\left(\frac{\partial T}{\partial y}\right)_{y=0}$

$q_{m}=-D\left(\frac{\partial C}{\partial y}\right)_{y=0}$

Applying the variable from equation (7), using equations $(14,-16)$ in (13) we obtain,

$\frac{1}{2} C_{f} \sqrt{R e_{x}}=\left(1+\frac{1}{\beta}\right) f^{\prime \prime}(0)$

$N u_{x} R e_{x}^{-\frac{1}{2}}=-\left(1+\frac{4}{3} N r\right) \theta^{\prime}(0)$
$S h_{x} R e_{x}^{-\frac{1}{2}}=-\phi^{\prime}(0)$

\section{5. CONFIRMATION OF NUMERICAL UPSHOTS}

The moderate bvp $4 \mathrm{c}$ with technique of shooting is applied to explain the highly nonlinear joined equations [8,9 \& 10] along with the associated boundary conditions [11]. Table.1 shows the assessment of present outcomes with that of previous outcomes, where we can observe exact agreement.

Table 1: Assessment values of $-f^{\prime \prime}(0)$ for various values of $M$ when $\beta=F^{*}=D=R=N=E_{1}=E=0$

\begin{tabular}{|l|l|l|l|l|}
\hline $\mathrm{M}$ & $\begin{array}{l}\text { Hayat et al. } \\
(2009)[31]\end{array}$ & $\begin{array}{l}\text { Mabood } \\
\text { and Das } \\
(2016)[32]\end{array}$ & $\begin{array}{l}\text { Mabood et } \\
\text { al. } \\
(2016)[33]\end{array}$ & $\begin{array}{l}\text { Present } \\
\text { results }\end{array}$ \\
\hline 0 & 1.0000 & 1.000008 & 1.000008 & 1.000007 \\
\hline 1 & 1.4142 & 1.4142 & 1.4142 & 1.41422 \\
\hline 5 & 2.4494 & 2.4494 & 2.4494 & 2.44941 \\
\hline 10 & 3.31662 & 3.31662 & 3.31662 & 3.31662 \\
\hline 50 & 7.14142 & 7.14142 & 7.14142 & 7.141422 \\
\hline 100 & 10.04987 & 10.04987 & 10.04987 & 10.04987 \\
\hline 500 & 22.38308 & 22.38380 & 22.38380 & 22.38381 \\
\hline 1000 & 31.63858 & 31.63858 & 31.63858 & 31.63858 \\
\hline
\end{tabular}

\section{GRAPHICAL ILLUSTRATIONS}

\subsection{Performance of $\beta, M$ and $E_{1}$ on $f^{\prime}(\eta), \theta(\eta), \varphi(\eta)$}

The effect of non - Newtonian rheology parameter $(\beta)$, magnetic field parameter $(M)$ and electrical field parameter $\left(E_{1}\right)$ can be observed from Fig. 2(a-f). $\beta$ accelerates fluid's plastic dynamic viscosity and resists the fluid flow, as a result gradual fall in velocity can be observed in Fig. 2(a). Advanced numerical values of $\beta$ increases wideness of the boundary layers in the fluid as a consequence there is gradual increase

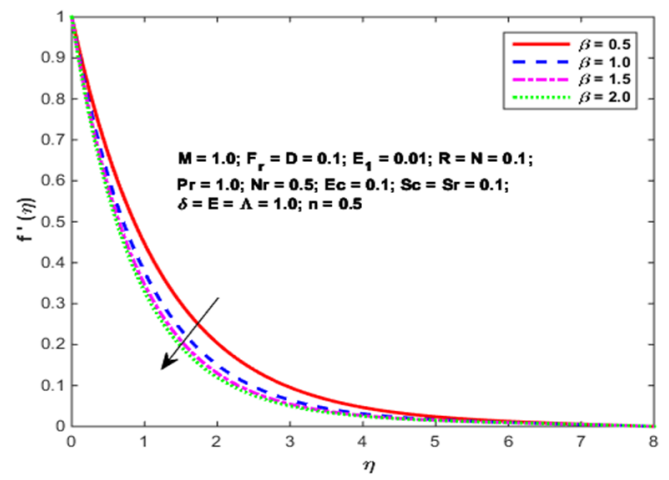

Fig. 2(a) Dominance of $\beta$ on $f^{\prime}(\eta)$

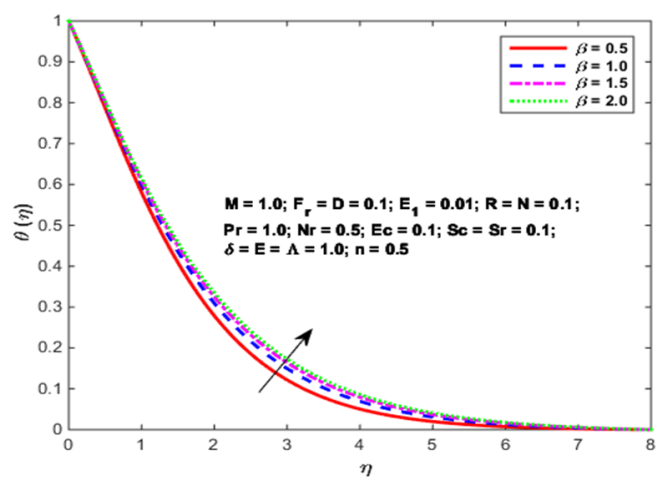

Fig. 2(b) Dominance of $\beta$ on $\theta(\eta)$ 


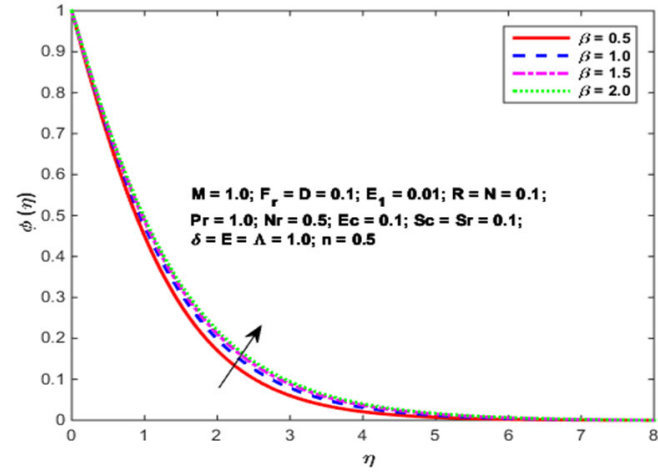

Fig. 2(c) Dominance of $\beta$ on $\phi(\eta)$

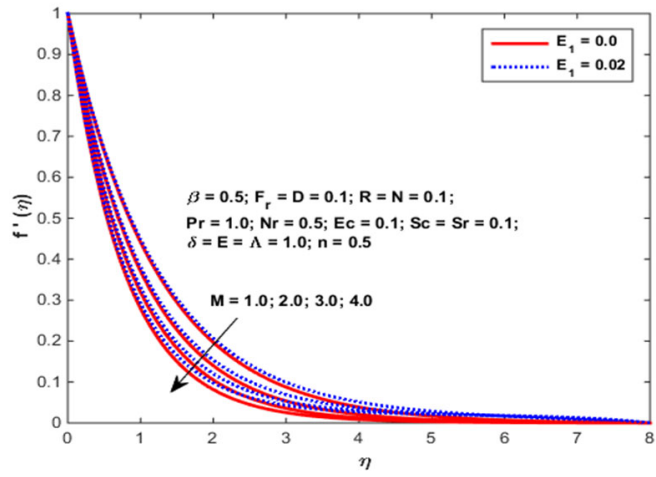

Fig. 2(d) Dominance of $\mathrm{M}$ and $E_{1}$ on $f^{\prime}(\eta)$

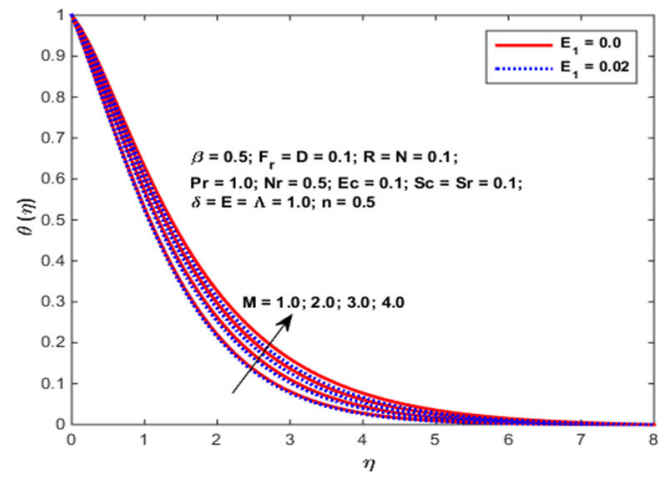

Fig. 2(e) Dominance of of $\mathrm{M}$ and $E_{1}$ on $\theta(\eta)$

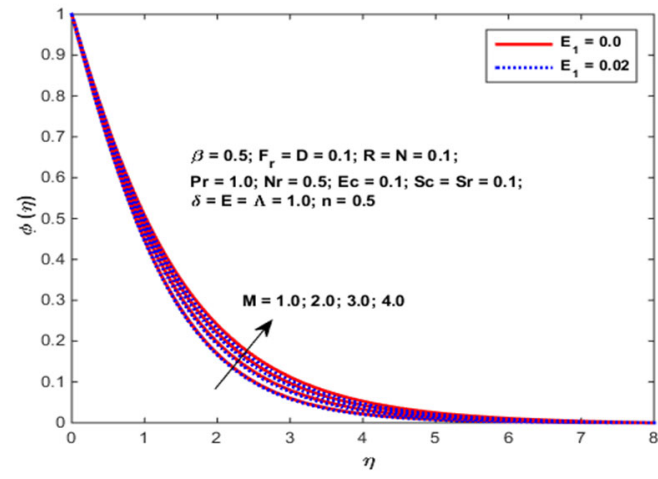

Fig. 2(f) Dominance of $M$ and $E_{1}$ on $\varphi(\eta)$

in temperature and concentration which is shown in Fig. 2(b) \& Fig. 2(c). From Figures. 2 (d-f) it is witnessed that as $M$ reaches higher values there is a quick reduction in velocity due to Lorentz force which compete against the motion of the fluid, at the same time there is gradual increment in both thermal and solutal boundary layers. Larger value of $E_{1}$ serves as accelerating force to overcome frictional resistance in the fluid and increases velocity, where there is reduction in temperature and species concentration due to increase in mass flux.

\subsection{Performance of $R, N, D, F r o n f^{\prime}(\eta), \theta(\eta), \varphi(\eta)$}

The influence of thermal buoyancy parameter (R), solutal buoyancy parameter $(\mathrm{N})$, permeability parameter $(\mathrm{D})$, and inertial parameter $(\mathrm{Fr})$ on $f^{\prime}(\eta), \theta(\eta), \phi(\eta)$ can be observed from Figures 3(a-l). Higher values of $\mathrm{R}$ accelerates velocity of the fluid where as temperature and concentration shows reverse effect for advanced values of R. Exactly similar effect on these gradients can be observed for $\mathrm{N}$. The velocity distribution shows a decreasing tendency for higher value of $\mathrm{D}$ and it shows reverse effect on both. It is observed that Fr decreases velocity profile and increases temperature and concentration profiles.

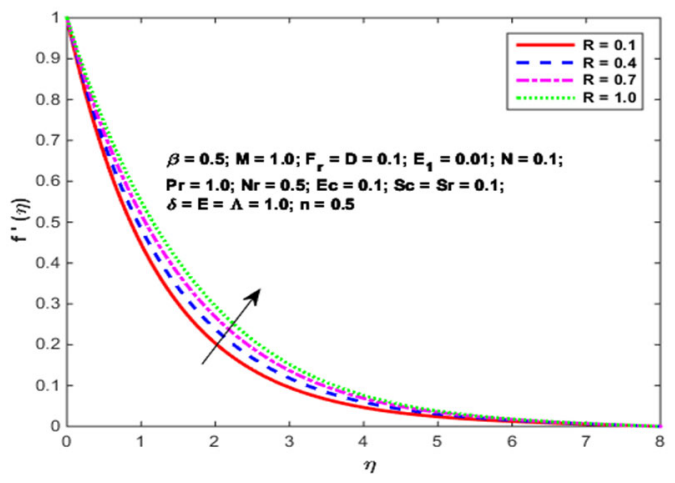

Fig. 3(a) Dominance of R on $f^{\prime}(\eta)$

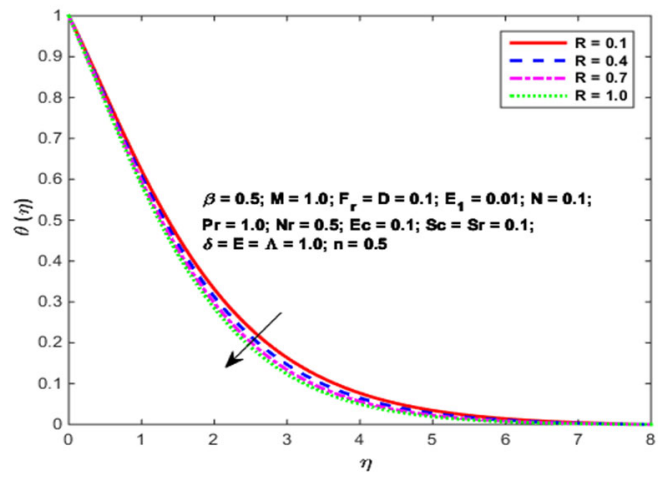

Fig. 3(b) Dominance of $\mathrm{R}$ on $\theta(\eta)$

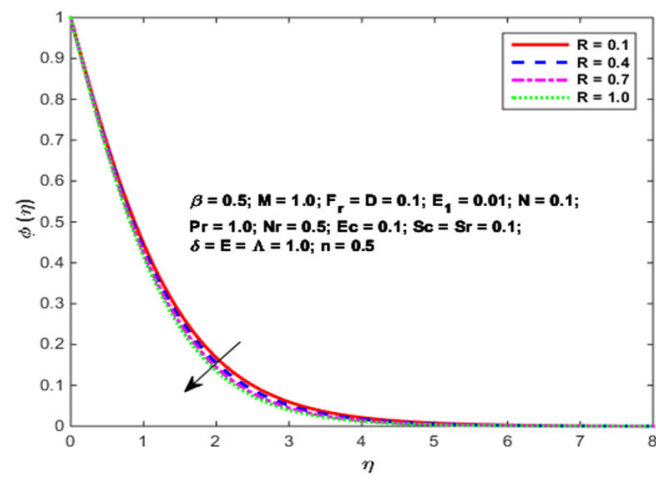

Fig. 3(c) Dominance of R on $\phi(\eta)$ 


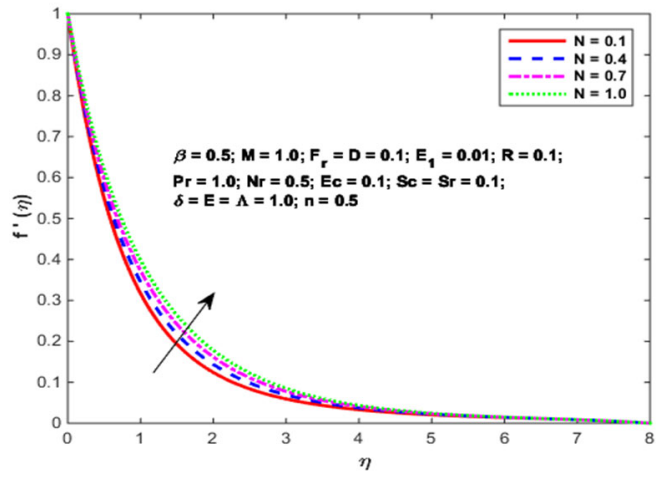

Fig. 3(d) Dominance of $\mathrm{N}$ on $f^{\prime}(\eta)$

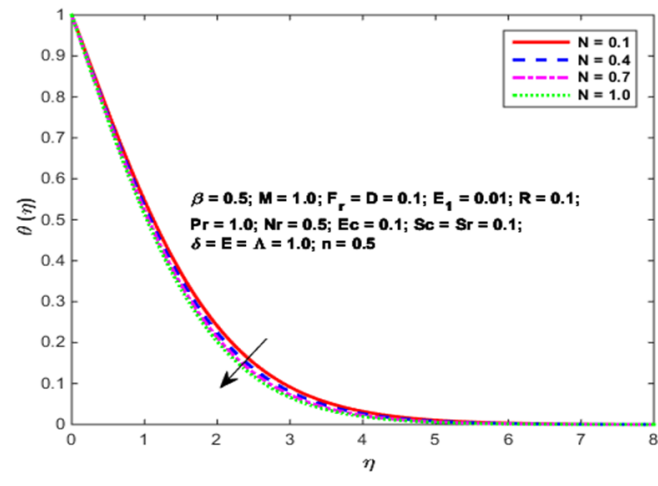

Fig. 3(e) Dominance of $\mathrm{N}$ on $\boldsymbol{\theta}(\boldsymbol{\eta})$

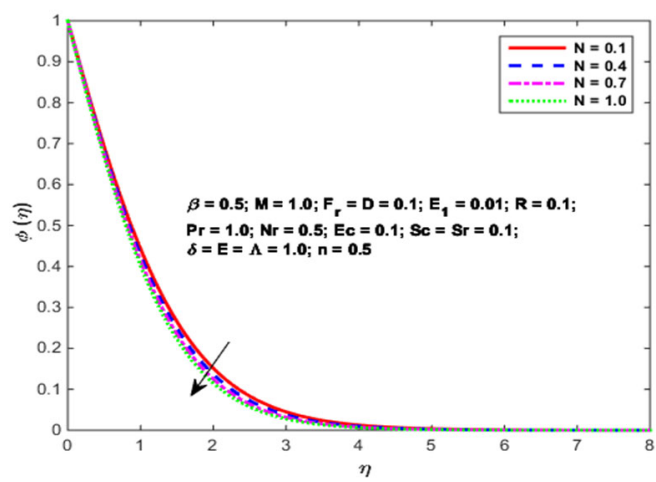

Fig 3(f). Dominance of $\mathrm{N}$ on $\phi(\eta)$

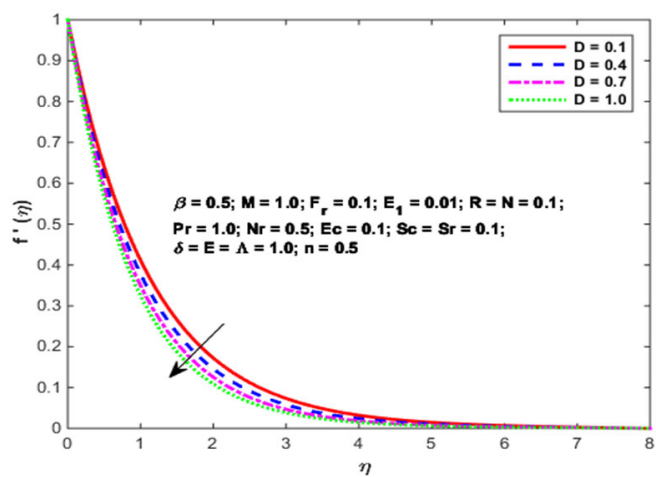

Fig. 3(g) Dominance of D on $f^{\prime}(\eta)$

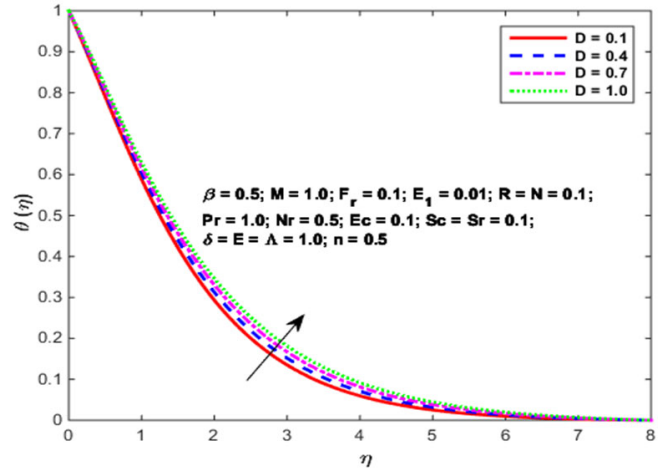

Fig. 3(h) Dominance of D on $\theta(\eta)$

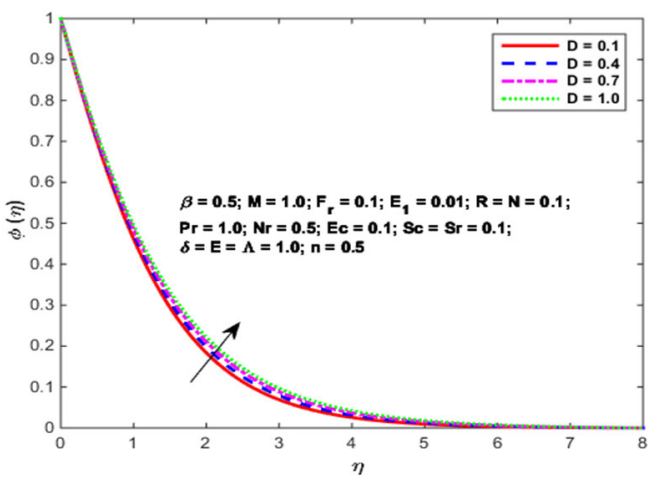

Fig. 3(i) Dominance of D on $\phi(\eta)$

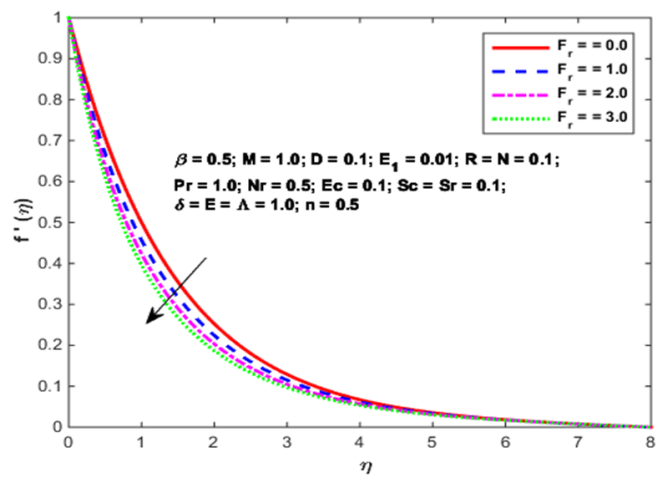

Fig. 3(j) Dominance of $F_{r}$ on $f^{\prime}(\eta)$

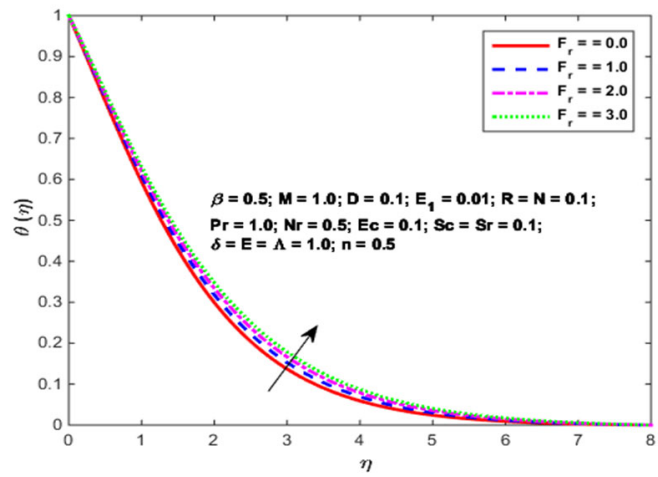

Fig. 3(k) Dominance of $F_{r}$ on $\theta(\eta)$ 


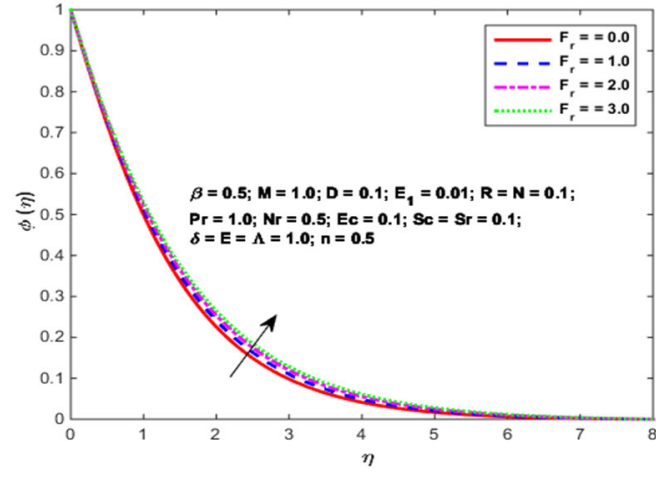

Fig. 3(I) Dominance of $F_{r}$ on $\phi(\eta)$

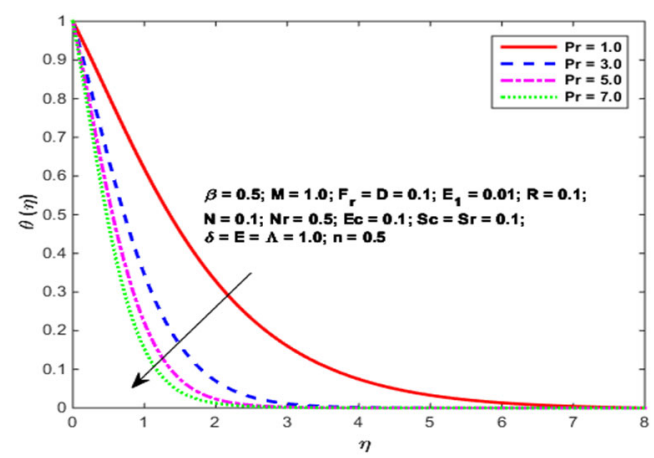

Fig. 4(a) Dominance of $\operatorname{Pr}$ on $\theta(\eta)$

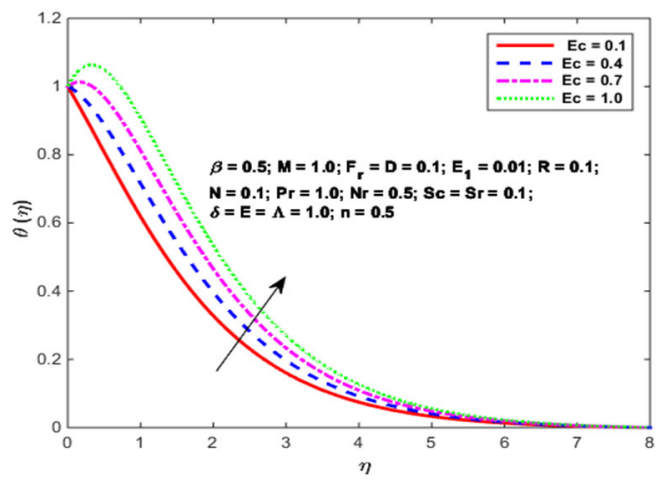

Fig. 4(b) Dominance of Ec on $\theta(\eta)$

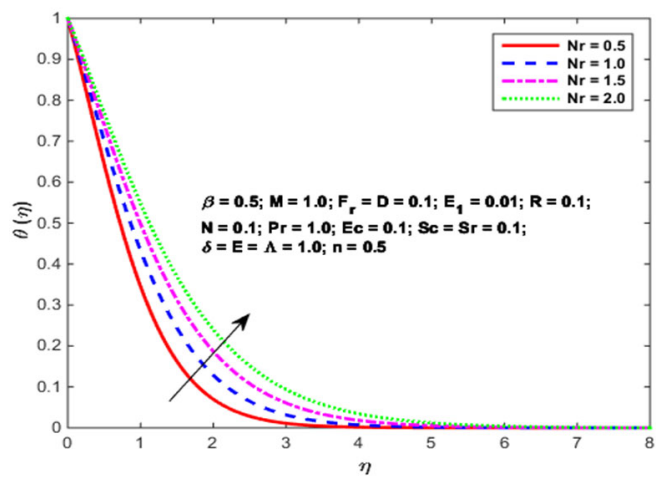

Fig. 4(c) Dominance of $\mathrm{Nr}$ on $\theta(\eta)$

\section{3 Performance of Pr, Ec, Nr on $\theta(\eta)$}

The performance of Prandtl number (Pr), Eckert number (Ec), thermal radiation parameter $(\mathrm{Nr})$ can be observed in Figures. 4(a-c). Comparing to conduction convection plays dominant role in boundary layer in transforming energy, this physical phenomena can be attributed from Fig. 4(a). For higher values of Pr more reduction in temperature is seen. Higher Ec values enhance temperature due to release of stored energy which is illustrated in Fig. 4(b). Larger value of $\mathrm{Nr}$ amplifies radiation and it leads to hike in temperature as shown in Fig. 4(c)

\subsection{Performance of $S c, S r, \Lambda, E$ and $n$ on $\varphi(\eta)$}

The performance for Schmidt number $(\mathrm{Sc})$, Soret number $(\mathrm{Sr})$, reaction rate parameter $(\Lambda)$, temperature difference parameter $(\delta)$, activation energy parameter (E) and fitted rate constant $(n)$ on species concentration are portrayed in Figures 5(a-f).Wideness of the solutal boundary layer becomes thinner when $\mathrm{Sc}$ is large as a result concentration reduces. Sr appreciable enhancement on concentration

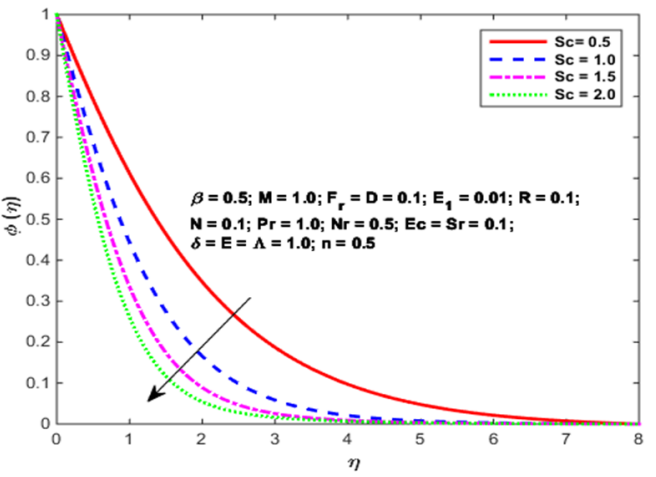

Fig. 5(a) Dominance of $S c$ on $\phi(\eta)$

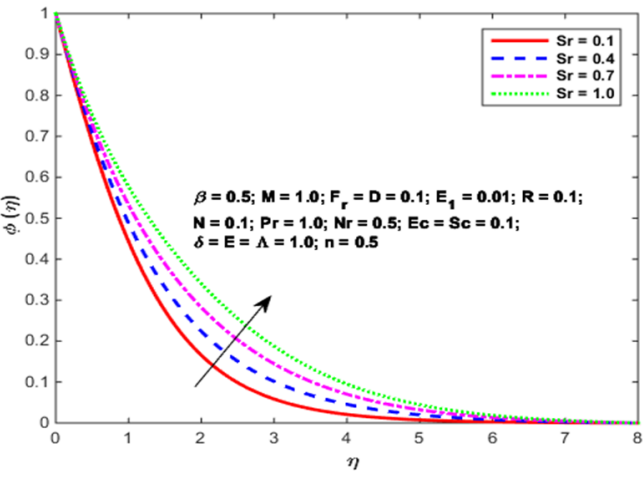

Fig. 5(b) Dominance of $S r$ on $\phi(\eta)$

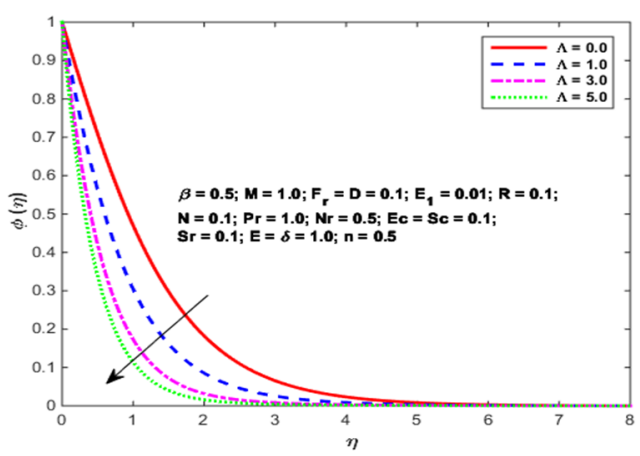

Fig. 5(c) Dominance of $\Lambda$ on $\phi(\eta)$ 


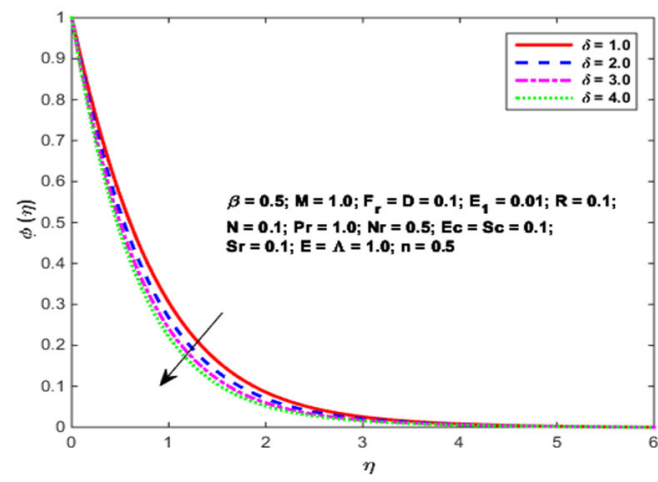

Fig. 5(d) Dominance of $\delta$ on $\phi(\eta)$

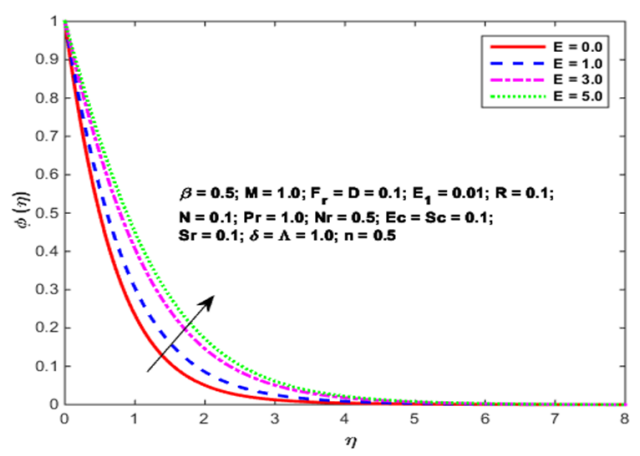

Fig. 5(e) Dominance of E on $\phi(\eta)$

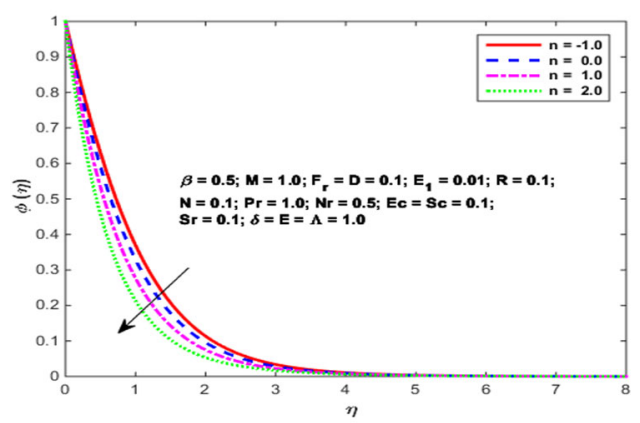

Fig. 5(f) Dominance of $\mathrm{n}$ on $\phi(\eta)$

distribution. $\delta$ Diminish concentration. As we improve the values of $\Lambda$ there is higher distructive amount of chemical reaction in the fluid and reduces concentration. The impact of $E$ and ' $n$ ' depicted in these graphs. It can be observed that enhancing value of $\mathrm{E}$ amplifies concentration, but fitted rate constant declines it.

\subsection{Influence of critical parameters on physical quantities}

Dominance of electrical field parameter $\left(\mathrm{E}_{1}\right)$ along with magnetic field parameter $(\mathrm{M})$ is portrayed in Fig. 6(a). When $M$ assumes higher values skin friction coefficient decreases and $\mathrm{E}_{1}$ shows opposite behavior, this is because of the Lorentz force produced due to the magnetic field parameter. Both thermal and solutal buoyancy parameters $(\mathrm{R}, \mathrm{N})$ are found to enhance skin friction which is depicted in Fig. 6(b). Both porous parameter and inertial parameter (D, Fr) decreases surface drag coefficient, as higher values of suction parameter reduce the force employed on the boundary layer of the fluid as illustrated in Fig. 6(c). From Fig. 6(d), it is noted that aggregate values of fitted rate constant ' $n$ ' increases the Sherwood number in very marginal difference. Whereas reaction rate parameter $(\Lambda)$ shows augmenting performance on it. Intensifying values of activation energy parameter (E) slows down the. It is pragmatic that high Prandtl number (Pr) increases Nusselt number. It is also seen that higher values of $\mathrm{Nr}$ has small influence on Nusselt number and Ec number decreases its value.

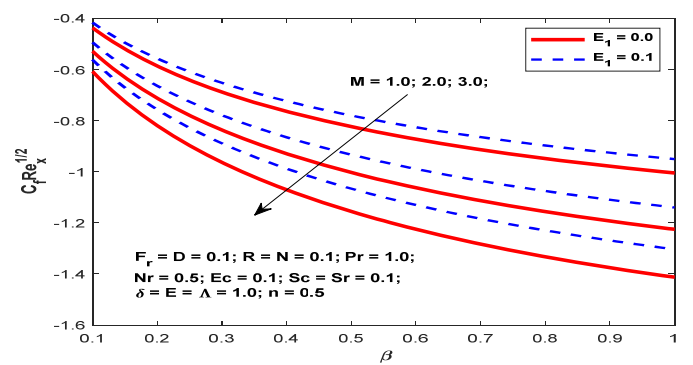

Fig. 6(a). Variation of $\mathrm{M}$, and $E_{1}$ on $C_{f} R e_{x}^{1 / 2}$

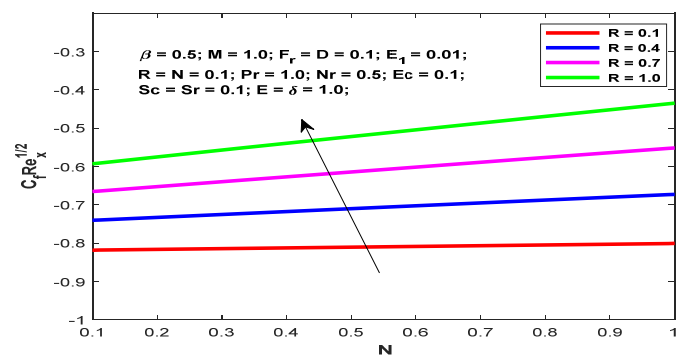

Fig. 6(b) Variation of $\mathrm{R}$ and $\mathrm{N}$ on $C_{f} R e_{x}^{1 / 2}$

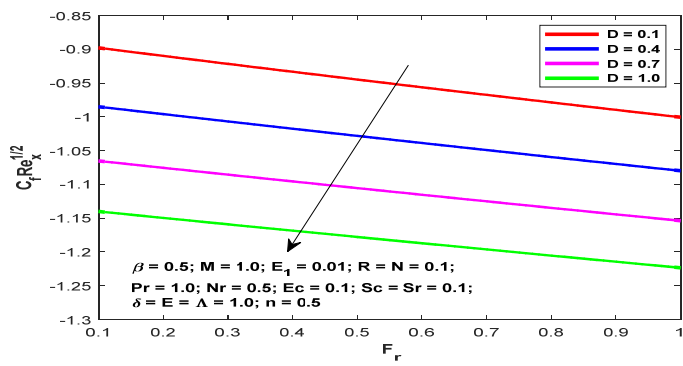

Fig. 6(c) Variation of $\mathrm{D}$ and $F_{r}$ on $C_{f} R e_{x}^{1 / 2}$

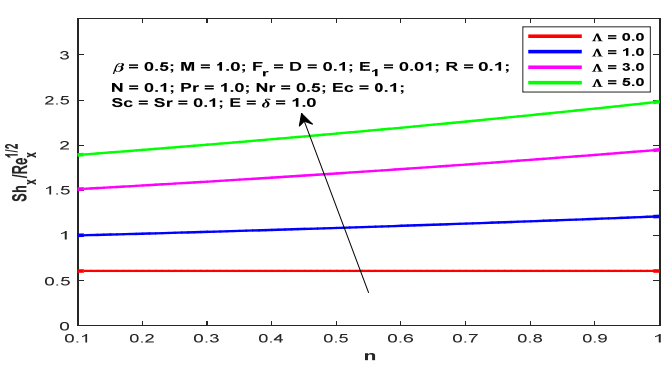

Fig. 6(d) Variation of $\Lambda$ and $\mathrm{n}$ on $S h_{x} R e_{x}^{-1 / 2}$

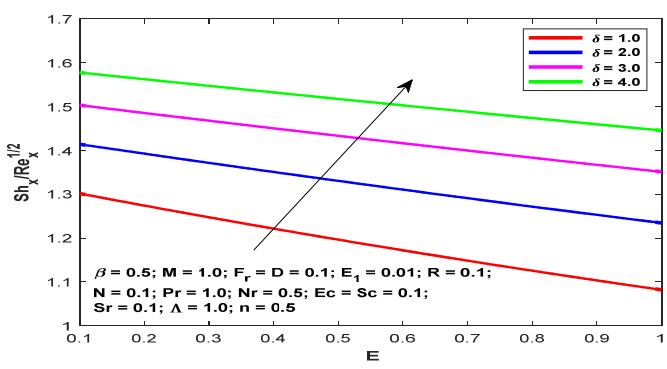

Fig. 6(f) Variation of $\delta$ and $E$ on $S h_{x} \operatorname{Re}_{x}^{-1 / 2}$ 


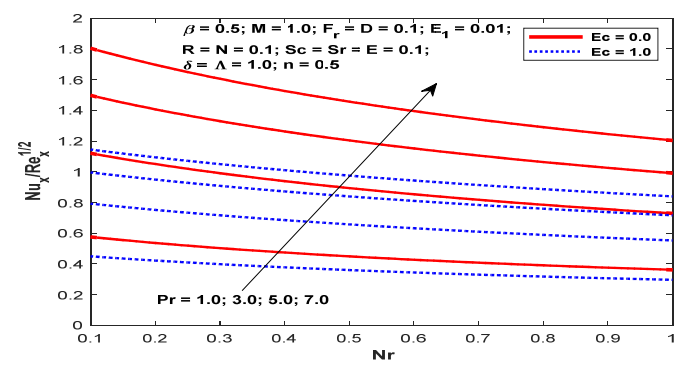

Fig. 6(g) Variation of $\mathrm{Pr}, \mathrm{Nr}$ and $\mathrm{Ec}$ on $N u_{x} R e_{x}^{-1 / 2}$

\section{CONCLUSIONS}

The present communication inspects the impact of Arrhenius activation energy along with chemical reaction of electrically conducting Casson fluid under the influence of transverse magnetic field, thermal diffusion induced due to porous stretching sheet. The impact of critical parameters on the flow configurations is graphically accessible using bvp $4 c$ shooting technique. The strategic consequences of the frame work are defined below.

$>$ The fluid velocity $f^{\prime}(\eta)$ enhanced for the progressive values of activation energy parameter E, Thermal buoyancy parameter R, Solutal buoyancy parameter $\mathrm{N}$, where conflicting tendency is noted for Non-Newtonian Rheology parameter $\beta$, magnetic parameter $\mathrm{M}$, and permeability parameter D.

$>$ Casson fluid temperature heightened for larger values of $\beta$, $\mathrm{M}, \mathrm{D}, \mathrm{Ec}, \mathrm{Nr}$ where as temperature decreased for higher values of $E_{1}, R, N$, Pr.

$>$ Enhancement in Schmidt number Sc, Chemical reaction parameter $\boldsymbol{\Lambda}$, Activation energy parameter E, temperature difference parameter $\delta$ produced thinner boundary layers with a reduction in concentration.

$>$ When fitted rate constant ' $n$ ' changes from $n=-1$ to $n=2$ a gradual reduction in concentration is observed.

$>$ Results of the analysis are found to be in an excellent with that of previous values.

\section{NOMENCLATURE}

$k^{*} \quad$ Absorption coefficient

$g \quad$ Acceleration due to gravity

$\bar{u} \quad$ Component of velocity along $\mathrm{x}$ - axis

$C_{\infty} \quad$ Concentration in the free stream

$C^{*} \quad$ Concentration of the fluid

$\bar{v} \quad$ Component of Velocity along $\mathrm{y}$ - axis

$C_{b} \quad$ Drag coefficient

$E_{0} \quad$ Electric field strength in transverse direction

$v \quad$ Kinematic viscosity

$B_{0} \quad$ Magnetic field strength in transverse direction

D Mass diffusivity

$T_{m} \quad$ Mean fluid temperature

$k_{p} \quad$ Permeability of the porous medium

$T_{\infty} \quad$ Temperature in the free stream

$T_{w} \quad$ Uniform temperature at the wall

$c_{p} \quad$ Specific heat at constant pressure

$T^{*} \quad$ Temperature of the fluid

$k \quad$ Thermal conductivity of the medium

$k_{T^{*}} \quad$ Thermal-diffusion ratio

$C_{w} \quad$ Uniform concentration at the wall
$\beta_{C} \quad$ Concentration expansion Coefficient

$\rho \quad$ Density of the fluid

$\mu \quad$ Dynamic viscosity of the fluid

$\sigma^{*} \quad$ Stefan-Boltzmann constant

$\beta_{T} \quad$ Thermal expansion Coefficient

\section{REFERENCES}

Arifuzzaman, S.M., Khan, M.S., Al-Mamun, A., Reza-E-Rabbi, S., Biswas, P., 2019, "Hydrodynamic Stability and Heat and Mass Transfer Flow Analysis of MHD Radiative Fourth-grade Fluid through Porous Plate with Chemical Reaction," Journal of King Saud University - Science, 31, 1388-1398.

https://doi.org/10.1016/j.jksus.2018.12.009

Arifuzzaman, S.M., Khan, M.S., Mehedi M. F. U., Rana, B.M J., Ahmmed S.F., 2018, "Chemically Reactive and Naturally Convective High-Speed MHD Fluid Flow through an Oscillatory Vertical PorousPlate with Heat and Radiation Absorption Effect," Engineering Science and Technology, an International Journal, 21(2), 215-228. https://doi.org/10.1016/j.jestch.2018.03.004

Casson, N. 1959, "A flow equation for pigment oil suspensions of printing ink type. In Rheology of Dispersed Systed," (Edited by C.C. Mill), 84-102, Pergamon Press, Oxford.

Chandra Sekhar, K.V., 2018, "Heat transfer analysis of second grade fluid over a stretching sheet through porous medium under the influence of chemical reaction parameter," International Journal of Mechanical and Production Engineering Research and Development, 8 (1), 605-612

https://doi.org/10.24247/ijmperdfeb201867

Charan Kumar, G., Jayarami Reddy, K., Ramakrishna, K., Narendradh Reddy M.,(2018). Non-uniform heat source/sink and joule heating effects on chemically radiative MHD mixed convective flow of micropolar fluid over a stretching sheet in porous medium. Defect and Diffusion Forum, 388, 281-302.

https://doi.org/10.4028/www.scientific.net/DDF.388.281

Dhanalakshmi, M., Jyothi, V., Jayarami Reddy, K., 2019, "Soret and Dufour Effects on MHD Convective Flow Past a Vertical Plate Through Porous Medium," Journal of Physics: Conference Series, 1344(1), PP.-.

https://doi.org/10.1088/1742-6596/1344/1/012008

Dharmaiah, G., Rani, Ch Baby., Vedavathi, N., Balamurugan, K. S., 2018, "heat and mass transfer on mhd fluid flow over a semi infinite flat plate with radiation absorption, heat source and diffusion thermo effect," Frontiers in heat and mass transfer, 11,6.

https://doi.org/10.5098/hmt.11.6

Eldabe, N. T. M., Salwa, M. G. E., 1995, "Heat transfer of MHD nonNewtonian Casson fluid flow between two rotating cylinder," $J$. Phys. Soc. Japan, 64, 41.

Fazal Mabood., Kalidas Das, 2019, "Outlining the impact of melting on MHD Casson fluid flow past a stretching sheet in a porous medium with radiation," Heliyon 5, e01216.

https://dx.doi.org/10.1016\%2Fj.heliyon.2019.e01216

Gayatri, M., Jayarami Reddy, K., Jayachandra Babu, M., 2020, “Slip fow of Carreau fuid over a slendering stretching sheet with viscous dissipation and Joule heating," SN Applied Sciences, 2:494.

https://doi.org/10.1007/s42452-020-2262-x 
Hari, R, Kataria., Harshad, R. Patel.,2018, "Heat and mass transfer in Magnetohydrodynamic (MHD) Casson fluid flow past over an oscillating vertical plate embedded in porous medium with ramped wall temperature," Propulsion and power research, 7(3), 257-267. https://doi.org/10.1016/j.jppr.2018.07.003

Hayata, T., Khan, A.A., Farhat Bibi., Farooq S. (2019). Activation energy and non- Darcy resistance in magneto peristalsis of Jeffrey material, Journal of Physics and chemistry of solids., Volume ,129, $155-161$.

https:///doi:10.1016/j.jpcs.2018.12.044.

Hayat, T., Hussain, Q., Javed, T., 2009, "The modified decomposition method and pade approximations for the MHD flow over a non-linear stretching sheet," Nonlinear Anal. Real World Appl, 10, 966-973.

https://doi.org/10.1016/j.nonrwa.2007.11.020

Hsiao, K. L., 2017, “To promote radiation electrical MHD activation energy thermal extrusion manufacturing system efficiency by using Carreau- Nanofluid with parameters control method," Energy 130, 486-499.

https://doi.org/10.1016/j.energy.2017.05.004

Irfan, M., Khan, W. A., Khan M., Mudassar Gulzar M., 2019, "Influence of Arrhenius activation energy in chemically reactive radiative flow of 3D Carreau nano fluid with non linear mixed convection," Journal of Physics and Chemistry of Solids, 125, 141-152. https://doi.org/10.1016/j.jpcs.2019.05.021

Krishna Y.H., Reddy G.V.R., Makinde O.D., 2018, "Chemical reaction effect on MHD flow of casson fluid with porous stretching sheet," Defect and Diffusion Forum, 389(), 100-109.

https://doi.org/10.4028/www.scientific.net/DDF.389.100

Konda, Jayarami Reddy., Madhusudhana, N. P., Konijeti, Ramakrishna., 2018, "MHD mixed convection flow of radiating and chemically reactive Casson nanofluid over a nonlinear permeable stretching sheet with viscous dissipation and heat source," multidiscipline modeling in materials and structures, 14(81) https://doi.org/ 10.1108/MMMS-10-2017-0127

Kumar D.R., Reddy K.J., Raju M.C. 2019)., "Unsteady MHD thermal diffusive and radiative fluid flow past a vertical porous plate with chemical reaction in slip flow regime," International Journal of Applied Mechanics and Engineering, 24(1), PP.117-129.

https://doi.org/10.2478/ijame-2019-0008

Maboob, F., Imtiaz, M., Alsaedi, A. and Hayat, T., 2016, "Unsteady convective boundary layer flow of fluid with nonlinear thermal radiation A numerical study," Int. J. Nonlinear Sciences and Numerical Simulation, 1-9.

https://doi.org/10.1515/ijnsns-2015-0153

Maboob, F. and Das, K., 2016, "Melting heat transfer on hydromagnetic flow of a nanofluid over a stretching sheet with radiation and second order slip," European Phys. J. Plus, 131(3), 1-12. https://doi.org/10.1140/epip/i2016-16003-1

Nagalakshmi, P. S, S., Vijaya, N., 2020, "MHD flow of Carreau Nanofluid explored using CNT over a nonlinear stretched sheet," Frontiers in Heat and Mass Transfer , 14, 4.

https://doi.org/10.5098/hmt.14.4

Naga Santoshi, P., Ramana Reddy, G.V., , P. Padma, P., 2020, "Numerical Scrutinization of Three Dimensional Casson Carreau Nano Fluid Flow," J. Appl. Comput. Mech, 6(3), 531-542.

https://doi.org/ 10.22055/JACM.2019.29377.1593
Sulochana, C., Poornima, M.,2019, "Unsteady MHD Casson fluid flow through vertical plate in the presence of Hall current," $S N$ Applied Sciences, 1:1626.

https://doi.org/10.1007/s42452-019-1656-0

Radha Madhavi, M., Vijaya, N., Nagesh,P.,2019, "Influence of magnetic field, heat radiation and external surface temperature on nanofluids with different base fluids in mixed convective flows over a vertical circular cylinder". International Journal of Innovative Technology and Exploring Engineering, 8(5), 497-504.

Ramana Murthy C.V., Kavitha K.R., Jhansi Rani K.,2019, "A mathematical model for increasing incidence oftuberculosis in poverty driven confined areas and measures for control," International Journal of Engineering and Advanced Technology, 9(1), PP.68506857.https://doi.org/10.35940/ijeat.A2987.109119

Reza-E-Rabbi, SK., Arifuzzaman, S. M., Tanmoy Sarkar., Shakhaoath Khan, Md., Sarder Firoz Ahmmed., 2020, "Explicit Finite Difference Analysis of an Unsteady MHD Flow of a Chemically Reacting Casson Fluid Past a Stretching Sheet with Brownian Motion and Thermophoresis Effects," Journal of King Saud University - Science, 32 (1), 690-701. https://doi.org/10.1016/j.jksus.2018.10.017

Sandhya A., Reddy G.V.R., Deekshitulu G.V.S.R., 2019,"Steady on MHD Heat and Mass Transfer Flow of an Inclined Porous Plate in the Presence of Radiation and Chemical Reaction," Journal of Physics: Conference Series, 1344(1), PP.https://doi.org/10.2478/ijame-2020-0036

Satish Kumar, D., Ramana Murthy, C.H.V., Anusha, S., Makinde, O.D., 2019, "Mixed convective flow of a visco elastic fluid between two porous parellel plates," International Journal of Recent Technology and Engineering, 8(3), PP.4830-4834.

https://doi.org/10.35940/ijrte.C6871.098319

Vedavathi N., Dharmaiah G., Balamurugan K.S., Prakash J. (2017), 'Heat transfer on mhd nanofluid flow over a semi infinite flat plate embedded in a porous medium with radiation absorption, heat source and diffusion thermo effect', Frontiers in Heat and Mass Transfer, 9(38), PP.

http://dx.doi.org/10.5098/hmt.9.38

Vijaya, N., Hari Krishna, Y., Kalyani, K., Reddy, G.V.R., 2018, “ Soret and radiation effects on an unsteady flow of a casson fluid through porous vertical channel with expansion and contraction," Frontiers in Heat and Mass Transfer,11, 19.

https://doi.org /10.5098/hmt.11.19

Vijaya, N., Venkata Ramana Reddy, G., Hara Krishna, Y., 2019, "Non-Aligned Stagnation Point Flow of a Casson Fluid Past a Stretching Sheet in a Doubly Stratified Medium," Fluid Dynamics \& Materials Processing, 15(3), 233-251.

https://doi.org /10.32604/fdmp.2019.03727

Vijaya, N., Sunil Babu, G., Vellanki Lakshmi, N., 2020, "influence of critical parameters on liquid thin film flow of casson nano fluid over elongated sheet under thermophorosis and brownian motion," Frontiers in Heat and Mass Transfer, 15, 23. https://doi.org /10.5098/hmt.15.23

Zeeshan, A., Shehad, N., Ellahi,R, 2018, "Analysis of activation energy in Couette - Poiseuille flow of nano fluid in the presence of chemical reaction and convective boundary conditions," Results in Physics, 8,502-512.

https://doi.org/10.1016/j.rinp.2017.12.024 